\title{
STRONG CONVERGENCE THEOREMS OF FUZZY MAPPINGS SATISFYING RATIONAL-TYPE INEQUALITY IN HILBERT SPACES
}

\author{
IMO KALU AGWU* AND DONATUS IKECHI IGBOKWE
}

\begin{abstract}
By using the idea of parallelogram law in a real Hilbert space, we provide some fuzzy common fixed point theorems for a pair of fuzzy mappings satisfying certain rational-type inequality condition. The results obtained in this paper complement, improve and generalise some recent results so far announced in literature.
\end{abstract}

\section{INTRODUCTION}

The famous Banach contraction principle [15] has been very indispensable in solving existence problems in different areas of mathematical analysis. Let $(Z, d)$ be a metric space and $\Gamma: Z \longrightarrow$ $Z$ a self-mapping on $Z$. For all $\xi, \omega \in Z$, define $\Gamma$ as

$$
d(\Gamma \xi, \Gamma \omega) \leq \beta d(\xi, \omega)
$$

and as

$$
d(\Gamma \xi, \Gamma \omega) \leq \alpha_{1} d(\xi, \omega)+\alpha_{2} d(\xi, \Gamma \xi)+\alpha_{3} d(\omega, \Gamma \omega)+\alpha_{4} d(\xi, \Gamma \omega)+\alpha_{5} d(\omega, \Gamma \xi),
$$

where $\alpha_{i}(i=1,2, \cdots, 5)$ are nonnegative real numbers. Note that $\Gamma$ is called a contraction if $\beta \in(0,1)$ and nonexpansive if $\beta=1$ in (1.1). Also, if $\sum_{i=1}^{5} \alpha_{i}<1$ and (1.2) is satisfied, then $\Gamma$ is called a contractive-type mapping. On the other hand, $\Gamma$ will be referred to as nonexpansivetype mapping if $\sum_{i=1}^{5} \alpha_{i}=1$ and (1.2) is satisfied. Approximate fixed point theorems for contractive (and contractive-type) and nonexpansive (and nonexpansive-type) mappings, a rich resource for solving problems in engineering and mathematical sciences, have been an area of major interest for researchers all over the world. In [13], Gregus introduced a nonexpansive-type single-valued mapping which satisfies (1.2) with $\alpha_{4}=\alpha_{5}=0$ and $\alpha_{1}<1$ in the setting of a real Hilbert space. Afterwards, different mappings satisfying different contractive conditions have been introduced and studied for the case of metric and Banach spaces; see, for example, [2][10], [12], [14] and the references therein.

Until 1965, different classes of objects encountered in the real physical world were believed to have a precise criteria of enlisting them in a group. However, uncertainty due to imprecision or vagueness is an essential part of physical formulation of real world phenomenon. In [11], Zader introduced the idea of fuzzy set (i.e., a set $X$ in which each member, say $z \in X$, is

Department of Mathematics, Micheal Okpara University of Agriculture, Umudike, Umuahia Abia State, Nigeria

*CORRESPONDING AUTHOR

E-mail addresses: agwuimo@gmail.com, igbokwedi@yahoo.com.

Key words and phrases. strong convergence; generalised contractive-type mapping; real Hilbert space; common fixed point; fuzzy set; fuzzy mapping; rational-type inequality.

Received 29/08/2021. 
assigned a membership grade $\nu(z)$ taking values in $[0,1]$ such that $\nu(z)=0$ corresponds to nonmembership, $0<\nu(z)<1$ to partial membership and $\nu(z)=1$ to full membership) as a means of addressing this challenge. Fuzzy set can, in fact, be regarded as a generalisation of classical set and has been created in order to provide a basis for a unified treatment of important problems from various branches of mathematical analysis. Subsequently, fuzzy set has been an integral part of many branches of science, social science and engineering.

In [1], using the concept of fuzzy set, Heilpern introduced a class of fuzzy mapping (which is more general than set-valued mapping) and obtained an approximate fixed point for fuzzy contraction mapping in a metric linear space. Following the result in [1], a lot of different results has found their place in the current literature; see, for example, [14], [16] and the references therein. While we continue to investigate fuzzy fixed point theorems in metric and Banach spaces, it is worthy to note that a little consideration has been made for the case of a real Hilbert space. On this note, in [10], using the contractive-type mapping

$$
\begin{aligned}
D^{2}\left(\Gamma_{1} \xi, \Gamma_{2} \omega\right) \leq & \alpha_{1}\|\xi, \omega\|^{2}+\alpha_{2} P_{\alpha}^{2}\left(\xi, \Gamma_{1} \xi\right)+\alpha_{3} P_{\alpha}^{2}\left(\omega, \Gamma_{2} \omega\right) \\
& +\alpha_{4}\left\{\frac{P_{\alpha}^{2}\left(\xi, \Gamma_{2} \omega\right)+P_{\alpha}^{2}\left(\omega, \Gamma_{1} \xi\right)}{1+P_{\alpha}^{2}\left(\xi, \Gamma_{2} \omega\right) P_{\alpha}^{2}\left(\omega, \Gamma_{1} \xi\right)}\right\},
\end{aligned}
$$

where $\Gamma_{1}, \Gamma_{2}: H \longrightarrow \mathcal{W}(H)$ are fuzzy mappings (and $\mathcal{W}(H)$ is as defined below), Saluja, Magarde and Jhade proved the theorem below:

Theorem 1.1. Let $H$ be a real Hilbert space, $\alpha_{1}, \alpha_{2}, \cdots, \alpha_{5}$ nonnegative numbers satisfying $\sum_{i=1}^{5} \alpha_{i}<1$ and $\Gamma_{1}, \Gamma_{2}: H \longrightarrow \mathcal{W}(H)$ satisfying (1.3). Then, there exists a point $z \in H$ such that $\{z\} \subset \Gamma_{1} \cap \Gamma_{2}$.

Motivated by the above results and the remarkable role plays by fuzzy sets in different disciplines of mathematics, economics, engineering and domain of words, we ask the following question:

Question 1.1. Is it possible to construct a rational-type inequality condition that is more general than the one defined by (1.3) and still obtain strong convergence results for a pair of fuzzy mappings?

Using the rich geometric structure of the real Hilbert space, in this paper, we provide an affirmative answer to Question 1.1.

To start with, we introduce the following rational-type inequality condition: Let $H$ be a real Hilbert space and $\Gamma_{1}, \Gamma_{2}: H \longrightarrow \mathcal{W}(H)$ a pair of fuzzy mappings satisfying

$$
\begin{aligned}
D^{2}\left(\Gamma_{1} x, \Gamma_{2} y\right) \leq & \beta_{1}\|x-y\|^{2}+\beta_{2} P_{\alpha}^{2}\left(x, \Gamma_{1} x\right)+\beta_{3} P_{\alpha}^{2}\left(y, \Gamma_{2} y\right)+\beta_{4}\left\{\frac{P_{\alpha}^{2}\left(y, \Gamma_{1} x\right)+P_{\alpha}^{2}\left(x, \Gamma_{2} y\right)}{1+P_{\alpha}^{2}\left(y, \Gamma_{1} x\right) P_{\alpha}^{2}\left(x, \Gamma_{2} y\right)}\right\} \\
& +\frac{\beta_{5}}{2}\left\{\left(P_{\alpha}^{2}\left(x, \Gamma_{1} x\right)+\frac{P_{\alpha}^{2}\left(y, \Gamma_{1} x\right)+P_{\alpha}^{2}\left(x, \Gamma_{2} y\right)}{1+P_{\alpha}^{2}\left(y, \Gamma_{1} x\right) P_{\alpha}^{2}\left(x, \Gamma_{2} y\right)}\right)\right.
\end{aligned}
$$




$$
\begin{aligned}
& \left.+\left(P_{\alpha}^{2}\left(y, \Gamma_{1} x\right)+\frac{P_{\alpha}^{2}\left(x, \Gamma_{1} x\right)+P_{\alpha}^{2}\left(x, \Gamma_{2} y\right)}{1+P_{\alpha}^{2}\left(x, \Gamma_{1} x\right) P_{\alpha}^{2}\left(x, \Gamma_{2} y\right)}\right)\right\} \\
& +\frac{\beta_{6}}{2}\left\{\left(P_{\alpha}^{2}\left(x, \Gamma_{1} x\right)+\frac{P_{\alpha}^{2}\left(y, \Gamma_{1} x\right)+P_{\alpha}^{2}\left(x, \Gamma_{2} y\right) P_{\alpha}^{2}\left(y, \Gamma_{2} y\right)}{1+P_{\alpha}^{2}\left(y, \Gamma_{1} x\right) P_{\alpha}^{2}\left(x, \Gamma_{2} y\right) P_{\alpha}^{2}\left(y, \Gamma_{1} y\right)}\right)\right. \\
& \left.+\left(P_{\alpha}^{2}\left(y, \Gamma_{1} x\right)+\frac{P_{\alpha}^{2}\left(x, \Gamma_{1} x\right)+P_{\alpha}^{2}\left(x, \Gamma_{2} y\right) P_{\alpha}^{2}\left(y, \Gamma_{2} y\right)}{1+P_{\alpha}^{2}\left(x, \Gamma_{1} x\right) P_{\alpha}^{2}\left(x, \Gamma_{2} y\right) P_{\alpha}^{2}\left(y, \Gamma_{2} y\right)}\right)\right\}
\end{aligned}
$$

for all $x, y \in H$ and for all $\alpha \in[0,1]$, where $\beta_{1}, \beta_{2}, \cdots, \beta_{6}$ are nonnegative numbers satisfying $\beta_{1}+\beta_{2}+\beta_{3}+\beta_{4}+\beta_{5}+\beta_{6}<1$.

Remark 1.1. Note that if $\beta_{5}=\beta_{6}=0$, then (1.4) reduces to (1.3). Also, if $\beta_{5}=\beta_{6}=0$ and $e=\frac{\beta_{4}}{2\left(1+P_{\alpha}^{2}\left(y, \Gamma_{1} x\right) P_{\alpha}^{2}\left(x, \Gamma_{2} y\right)\right)}$, then (1.4) will reduce to the contractive-type mapping defined in [9].

\section{PRELIMINARY}

Throughout the remaining sections, $\mathbb{R}, \mathbb{N}$ and $H$ will denote the set of real numbers, the set of natural numbers and a real Hilbert space, respectively.

Let $I=[0,1], H$ a real Hilbert space and $\mathcal{F}(H)$ a collection of fuzzy sets in $H$. A fuzzy set $\eta$ of $H$ is defined by its membership function $\eta(x)$, which is a mapping from $H$ into $I$. For any $\eta \in \mathcal{F}(H)$ and $\alpha \in[0,1]$, the $\alpha$-level of $\eta$ is defined as

$$
[\eta]_{\alpha}=\{x \in H: \eta(x) \geq \alpha\}
$$

where $\alpha \in(0,1)$ and denote the support $[\eta]_{0}$ of $\eta$ by the closure of the union of $[\eta]_{\alpha}$ for $0 \leq \alpha \leq 1$. Let $\mathcal{W}(H)$ be the subcollection of all approximate quantities of $\mathcal{F}(H)$. In what follows, we collect some useful definitions and lemmas which will be helpful in order to obtain our results.

Definition 2.1. A fuzzy set $\eta$ of $H$ is called an approximate quantity if and only if its $\alpha$-level set is a compact convex subset of $H$ for each $\alpha \in[0,1]$ and $\sup _{x \in H} \eta(x)=1$.

Definition 2.2. Let $Q, R \in \mathcal{W}(H)$ and $\alpha \in[0,1]$, then

(i) $P_{\alpha}(Q, R)=\inf _{x \in Q_{\alpha}, y \in R_{\alpha}}\|x-y\|$;

(ii) $D_{\alpha}(Q, R)=\max \left\{\sup _{x \in A_{\alpha}} d(x, B x), \sup _{x \in B_{\alpha}} d(x, A x)\right\}$;

(iii) $D(Q, R)=\sup _{\alpha} D_{\alpha}(Q, R)$ and

(iv) $P(Q, R)=\sup _{\alpha} P_{\alpha}(Q, R)$,

where $P_{\alpha}(Q, R), D_{\alpha}(Q, R)$ and $D(Q, R)$ denote $\alpha$-space, $\alpha$-distance between $Q$ and $R$ and distance between $Q$ and $R$, respectively. Note that for any $\alpha, P_{\alpha}$ is a nondecreasing continuous function of $\alpha$.

Definition 2.3. Let $Q, R \in \mathcal{W}(H)$. Then, $Q$ is said to be more accurate than $R$ (denoted as $Q \subset R$ ) if and only if $Q s<R s$ for each $s \in H$. The relation $\subset$ induces a partial ordering on $\mathcal{W}(H)$. 
Definition 2.4. Let $\mathcal{F}: H \longrightarrow \mathcal{W}(H)$ be a fuzzy mapping (i.e., $\mathcal{F}(x) \in \mathcal{W}(H)$ for each $x \in H)$. A point $x \in H$ is called a fixed point of $\mathcal{F}$ if $\{x\} \subset \mathcal{F}(x)$, where $\{x\}$ is the fuzzy set with membership function equal to the characteristic function.

Lemma 2.1. Let $x \in H, Q \in \mathcal{W}(H)$ and $\{x\}$ be a fuzzy set with membership function equal to the characteristic function of the set $\{x\}$. If $\{x\} \subset Q$, then $P_{\alpha}(x, Q)=0$ for each $\alpha \in[0,1]$.

Lemma 2.2. $P_{\alpha}(x, Q) \leq\|x-y\|+P_{\alpha}(y, Q)$, for any $x, y \in H$.

Lemma 2.3. If $\left\{x_{0}\right\} \subset Q$, then $P_{\alpha}\left(x_{0}, R\right) \leq D_{\alpha}(Q, R)$ for each $R \in \mathcal{W}(H)$.

\section{Main Results}

Theorem 3.1. Let $H$ be a Hilbert space and $\Gamma_{1}, \Gamma_{2}: H \longrightarrow \mathcal{W}(H)$ a pair of fuzzy mappings satisfying

$$
\begin{aligned}
D^{2}\left(\Gamma_{1} x, \Gamma_{2} y\right) \leq & \beta_{1}\|x-y\|^{2}+\beta_{2} P_{\alpha}^{2}\left(x, \Gamma_{1} x\right)+\beta_{3} P_{\alpha}^{2}\left(y, \Gamma_{2} y\right)+\beta_{4}\left\{\frac{P_{\alpha}^{2}\left(y, \Gamma_{1} x\right)+P_{\alpha}^{2}\left(x, \Gamma_{2} y\right)}{1+P_{\alpha}^{2}\left(y, \Gamma_{1} x\right) P_{\alpha}^{2}\left(x, \Gamma_{2} y\right)}\right\} \\
& +\frac{\beta_{5}}{2}\left\{\left(P_{\alpha}^{2}\left(x, \Gamma_{1} x\right)+\frac{P_{\alpha}^{2}\left(y, \Gamma_{1} x\right)+P_{\alpha}^{2}\left(x, \Gamma_{2} y\right)}{1+P_{\alpha}^{2}\left(y, \Gamma_{1} x\right) P_{\alpha}^{2}\left(x, \Gamma_{2} y\right)}\right)\right. \\
& \left.+\left(P_{\alpha}^{2}\left(y, \Gamma_{1} x\right)+\frac{P_{\alpha}^{2}\left(x, \Gamma_{1} x\right)+P_{\alpha}^{2}\left(x, \Gamma_{2} y\right)}{1+P_{\alpha}^{2}\left(x, \Gamma_{1} x\right) P_{\alpha}^{2}\left(x, \Gamma_{2} y\right)}\right)\right\} \\
& +\frac{\beta_{6}}{2}\left\{\left(P_{\alpha}^{2}\left(x, \Gamma_{1} x\right)+\frac{P_{\alpha}^{2}\left(y, \Gamma_{1} x\right)+P_{\alpha}^{2}\left(x, \Gamma_{2} y\right) P_{\alpha}^{2}\left(y, \Gamma_{2} y\right)}{1+P_{\alpha}^{2}\left(y, \Gamma_{1} x\right) P_{\alpha}^{2}\left(x, \Gamma_{2} y\right) P_{\alpha}^{2}\left(y, \Gamma_{1} y\right)}\right)\right. \\
& \left.+\left(P_{\alpha}^{2}\left(y, \Gamma_{1} x\right)+\frac{P_{\alpha}^{2}\left(x, \Gamma_{1} x\right)+P_{\alpha}^{2}\left(x, \Gamma_{2} y\right) P_{\alpha}^{2}\left(y, \Gamma_{2} y\right)}{1+P_{\alpha}^{2}\left(x, \Gamma_{1} x\right) P_{\alpha}^{2}\left(x, \Gamma_{2} y\right) P_{\alpha}^{2}\left(y, \Gamma_{2} y\right)}\right)\right\}
\end{aligned}
$$

for all $x, y \in H$ and for all $\alpha \in[0,1]$, where $\beta_{1}, \beta_{2}, \cdots, \beta_{6}$ are nonnegative numbers satisfying $\beta_{1}+\beta_{2}+\beta_{3}+\beta_{4}+\beta_{5}+\beta_{6}<1$. Then, there exists a point $x^{\star} \in H$ such that $\left\{x^{\star}\right\} \in \Gamma_{1} x^{\star} \cap \Gamma_{2} x^{\star}$.

Proof. Let $x_{0} \in H$ and $\left\{x_{1}\right\} \subset \Gamma_{1}\left(x_{0}\right)$. Then, there exists $x_{2} \in H$ such that $\left\{x_{2}\right\} \subset \Gamma_{1}\left(x_{1}\right)$ and $\left\|x_{1}-x_{2}\right\| \leq D_{1}\left(\Gamma_{1}\left(x_{0}\right), \Gamma_{2}\left(x_{1}\right)\right)$. Also, we can find $x_{3} \in H$ such that $\left\{x_{3}\right\} \subset \Gamma_{1}\left(x_{2}\right)$ and $\left\|x_{2}-x_{3}\right\| \leq D_{1}\left(\Gamma_{1}\left(x_{1}\right), \Gamma_{2}\left(x_{2}\right)\right)$. Continuing in this way, we would have a sequence $\left\{x_{n}\right\} \in H$ such that

$$
\left\{x_{1}\right\} \subset \Gamma_{1}\left(x_{0}\right),\left\{x_{2}\right\} \subset \Gamma_{2}\left(x_{1}\right), \cdots\left\{x_{2 n-1}\right\} \subset \Gamma_{1}\left(x_{2 n-2}\right),\left\{x_{2 n}\right\} \subset \Gamma_{2}\left(x_{2 n-1}\right)
$$

and

$$
\left\|x_{j}-x_{j+1}\right\| \leq D_{1}\left(\Gamma_{1}\left(x_{j-1}\right), \Gamma_{2}\left(x_{i}\right)\right), i=1,2, \cdots
$$


Now, using (3.1), Lemma 2.3, parallelogram law and the fact that $(a-b)^{2} \leq a^{2}+b^{2}$, we estimate as follows:

$$
\begin{aligned}
& \left\|x_{2 n-1}-x_{2 n}\right\|^{2} \\
& \leq D_{1}^{2}\left(\Gamma_{1}\left(x_{2 n-2}\right), \Gamma_{2}\left(x_{2 n-1}\right)\right) \leq D^{2}\left(\Gamma_{1}\left(x_{2 n-2}\right), \Gamma_{2}\left(x_{2 n-1}\right)\right) \\
& \leq \beta_{1}\left\|x_{2 n-2}-x_{2 n-1}\right\|^{2}+\beta_{2} P_{\alpha}^{2}\left(x_{2 n-2}, \Gamma_{1}\left(x_{2 n-2}\right)\right)+\beta_{3} P_{\alpha}^{2}\left(x_{2 n-1}, \Gamma_{2}\left(x_{2 n-1}\right)\right) \\
& +\beta_{4}\left\{\frac{P_{\alpha}^{2}\left(x_{2 n-1}, \Gamma_{1}\left(x_{2 n-2}\right)\right)+P_{\alpha}^{2}\left(x_{2 n-2}, \Gamma_{2}\left(x_{2 n-1}\right)\right)}{1+P_{\alpha}^{2}\left(x_{2 n-1}, \Gamma_{1}\left(x_{2 n-2}\right)\right) P_{\alpha}^{2}\left(x_{2 n-2}, \Gamma_{2}\left(x_{2 n-1}\right)\right)}\right\} \\
& +\frac{\beta_{5}}{2}\left\{\left(P_{\alpha}^{2}\left(x_{2 n-2}, \Gamma_{1}\left(x_{2 n-2}\right)\right)+\frac{P_{\alpha}^{2}\left(x_{2 n-1}, \Gamma_{1}\left(x_{2 n-2}\right)\right)+P_{\alpha}^{2}\left(x_{2 n-2}, \Gamma_{2}\left(x_{2 n-1}\right)\right)}{1+P_{\alpha}^{2}\left(x_{2 n-1}, \Gamma_{1}\left(x_{2 n-2}\right)\right) P_{\alpha}^{2}\left(x_{2 n-2}, \Gamma_{2}\left(x_{2 n-1}\right)\right)}\right)\right. \\
& \left.+\left(P_{\alpha}^{2}\left(x_{2 n-1}, \Gamma_{2}\left(x_{2 n-1}\right)\right)+\frac{P_{\alpha}^{2}\left(x_{2 n-2}, \Gamma_{1}\left(x_{2 n-2}\right)\right)+P_{\alpha}^{2}\left(x_{2 n-1}, \Gamma_{1}\left(x_{2 n-2}\right)\right)}{1+P_{\alpha}^{2}\left(x_{2 n-2}, \Gamma_{1}\left(x_{2 n-2}\right)\right) P_{\alpha}^{2}\left(x_{2 n-1}, \Gamma_{1}\left(x_{2 n-2}\right)\right)}\right)\right\} \\
& +\frac{\beta_{6}}{2}\left\{\left(P_{\alpha}^{2}\left(x_{2 n-2}, \Gamma_{1}\left(x_{2 n-2}\right)\right)\right.\right. \\
& \left.+\frac{P_{\alpha}^{2}\left(x_{2 n-2}, \Gamma_{2}\left(x_{2 n-1}\right)\right)+P_{\alpha}^{2}\left(x_{2 n-1}, \Gamma_{1}\left(x_{2 n-2}\right)\right) P_{\alpha}^{2}\left(x_{2 n-1}, \Gamma_{2}\left(x_{2 n-1}\right)\right)}{1+P_{\alpha}^{2}\left(x_{2 n-2}, \Gamma_{2}\left(x_{2 n-1}\right)\right) P_{\alpha}^{2}\left(x_{2 n-1}, \Gamma_{1}\left(x_{2 n-2}\right)\right) P_{\alpha}^{2}\left(x_{2 n-1}, \Gamma_{1}\left(x_{2 n-1}\right)\right)}\right) \\
& +\left(P_{\alpha}^{2}\left(x_{2 n-2}, \Gamma_{2}\left(x_{2 n-1}\right)\right)\right. \\
& \left.\left.\frac{P_{\alpha}^{2}\left(x_{2 n-2}, \Gamma_{1}\left(x_{2 n-2}\right)\right)+P_{\alpha}^{2}\left(x_{2 n-1}, \Gamma_{2}\left(x_{2 n-2}\right)\right) P_{\alpha}^{2}\left(x_{2 n-1}, \Gamma_{2}\left(x_{2 n-1}\right)\right)}{1+P_{\alpha}^{2}\left(x_{2 n-2}, \Gamma_{1}\left(x_{2 n-2}\right)\right) P_{\alpha}^{2}\left(x_{2 n-1}, \Gamma_{2}\left(x_{2 n-2}\right)\right) P_{\alpha}^{2}\left(x_{2 n-1}, \Gamma_{2}\left(x_{2 n-1}\right)\right)}\right)\right\} \\
& \leq \beta_{1}\left\|x_{2 n-2}-x_{2 n-1}\right\|^{2}+\beta_{2}\left\|x_{2 n-2}-x_{2 n-1}\right\|^{2}+\beta_{3}\left\|x_{2 n-1}-x_{2 n}\right\|^{2} \\
& +\beta_{4}\left\{\frac{\left\|x_{2 n-1}-x_{2 n-1}\right\|^{2}+\left\|x_{2 n-2}-x_{2 n}\right\|^{2}}{1+\left\|x_{2 n-1}-x_{2 n-1}\right\|^{2}\left\|x_{2 n-2}-x_{2 n}\right\|^{2}}\right\} \\
& +\frac{\beta_{5}}{2}\left\{\left(\left\|x_{2 n-2}-x_{2 n-2}\right\|^{2}+\frac{\left\|x_{2 n-1}-x_{2 n-1}\right\|^{2}+\left\|x_{2 n-2}-x_{2 n}\right\|^{2}}{1+\left\|x_{2 n-1}-x_{2 n-1}\right\|^{2}\left\|x_{2 n-2}-x_{2 n-1}\right\|^{2}}\right)\right. \\
& \left.+\left(\left\|x_{2 n-1}-x_{2 n}\right\|^{2}+\frac{\left\|x_{2 n-2}-x_{2 n-1}\right\|^{2}+\left\|x_{2 n-1}-x_{2 n-1}\right\|^{2}}{1+\left\|x_{2 n-2}-x_{2 n-1}\right\|^{2}\left\|x_{2 n-1}-x_{2 n-1}\right\|^{2}}\right)\right\} \\
& +\frac{\beta_{6}}{2}\left\{\left(\left\|x_{2 n-2}-x_{2 n-1}\right\|^{2} x+\frac{\left\|x_{2 n-2}-x_{2 n}\right\|^{2}+\left\|x_{2 n-1}-x_{2 n-1}\right\|^{2}\left\|x_{2 n-1}-x_{2 n}\right\|^{2}}{\left.\left.1+\left\|x_{2 n-2}-2 n\right\|^{2}\left\|x_{2 n-1}-x_{2 n-1}\right\|^{2} \| x_{2 n-1}-x_{2 n}\right)\right)}\right)\right. \\
& \left.+\left(\left\|x_{2 n-2}-x_{2 n}\right\|^{2}+\frac{\left\|x_{2 n-2}-x_{2 n-1}\right\|^{2}+\left\|x_{2 n-1}-x_{2 n-1}\right\|^{2}\left\|2 n-1-x_{2 n}\right\|^{2}}{1+\left\|x_{2 n-2}-x_{2 n-1}\right\|^{2}\left\|x_{2 n-1}-x_{2 n-1}\right\|^{2}\left\|x_{2 n-1}-x_{2 n}\right\|^{2}}\right)\right\} \\
& =\beta_{1}\left\|x_{2 n-2}-x_{2 n-1}\right\|^{2}+\beta_{2}\left\|x_{2 n-2}-x_{2 n-1}\right\|^{2}+\beta_{3}\left\|x_{2 n-1}-x_{2 n}\right\|^{2}+\beta_{4}\left\|x_{2 n-2}-x_{2 n}\right\|^{2} \\
& +\frac{\beta_{5}}{2}\left(2\left\|x_{2 n-2}-x_{2 n-1}\right\|^{2}+2\left\|x_{2 n-2}-x_{2 n}\right\|^{2}\right)+\frac{\beta_{6}}{2}\left(2\left\|x_{2 n-2}-x_{2 n-1}\right\|^{2}+2\left\|x_{2 n-2}-x_{2 n}\right\|^{2}\right) \\
& =\beta_{1}\left\|x_{2 n-2}-x_{2 n-1}\right\|^{2}+\beta_{2}\left\|x_{2 n-2}-x_{2 n-1}\right\|^{2}+\beta_{3}\left\|x_{2 n-1}-x_{2 n}\right\|^{2}+\beta_{4}\left\|x_{2 n-2}-x_{2 n}\right\|^{2} \\
& +\frac{\beta_{5}}{2}\left(\left\|x_{2 n-2}-x_{2 n-1}+x_{2 n-2}-x_{2 n}\right\|^{2}+\left\|x_{2 n-2}-x_{2 n-1}-\left(x_{2 n-2}-x_{2 n}\right)\right\|^{2}\right) \\
& +\frac{\beta_{6}}{2}\left(\left\|x_{2 n-2}-x_{2 n-1}+x_{2 n-2}-x_{2 n}\right\|^{2}+\left\|x_{2 n-2}-x_{2 n-1}-\left(x_{2 n-2}-x_{2 n}\right)\right\|^{2}\right) \\
& =\beta_{1}\left\|x_{2 n-2}-x_{2 n-1}\right\|^{2}+\beta_{2}\left\|x_{2 n-2}-x_{2 n-1}\right\|^{2}+\beta_{3}\left\|x_{2 n-1}-x_{2 n}\right\|^{2}+\beta_{4}\left\|x_{2 n-2}-x_{2 n}\right\|^{2} \\
& +\frac{\beta_{5}}{2}\left(\left\|x_{2 n-2}-x_{2 n-1}-\left(x_{2 n}-x_{2 n-2}\right)\right\|^{2}+\left\|x_{2 n}-x_{2 n-2}\right\|^{2}\right) \\
& +\frac{\beta_{6}}{2}\left(\left\|x_{2 n-2}-x_{2 n-1}-\left(x_{2 n}-x_{2 n-2}\right)\right\|^{2}+\left\|x_{2 n}-x_{2 n-2}\right\|^{2}\right)
\end{aligned}
$$




$$
\begin{aligned}
\leq & \beta_{1}\left\|x_{2 n-2}-x_{2 n-1}\right\|^{2}+\beta_{2}\left\|x_{2 n-2}-x_{2 n-1}\right\|^{2}+\beta_{3}\left\|x_{2 n-1}-x_{2 n}\right\|^{2}+\beta_{4}\left\|x_{2 n-2}-x_{2 n}\right\|^{2} \\
& +\frac{\beta_{5}}{2}\left(\left\|x_{2 n-2}-x_{2 n-1}\right\|^{2}+\left\|x_{2 n}-x_{2 n-2}\right\|^{2}+\left\|x_{2 n}-x_{2 n-2}\right\|^{2}\right) \\
& +\frac{\beta_{6}}{2}\left(\left\|x_{2 n-2}-x_{2 n-1}\right\|^{2}+\left\|x_{2 n}-x_{2 n-2}\right\|^{2}+\left\|x_{2 n}-x_{2 n-2}\right\|^{2}\right) \\
= & \left(\beta_{1}+\beta_{2}+\frac{\beta_{5}}{2}+\frac{\beta_{6}}{2}\right)\left\|x_{2 n-2}-x_{2 n-1}\right\|^{2}+\left(\beta_{3}+\frac{\beta_{5}}{2}+\frac{\beta_{6}}{2}\right)\left\|x_{2 n-1}-x_{2 n}\right\|^{2} \\
& +\left(\beta_{4}+\frac{\beta_{5}}{2}+\frac{\beta_{6}}{2}\right)\left\|x_{2 n-2}-x_{2 n-1}-\left(x_{2 n}-x_{2 n-1}\right)\right\|^{2} \\
\leq & \left(\beta_{1}+\beta_{2}+\frac{\beta_{5}}{2}+\frac{\beta_{6}}{2}\right)\left\|x_{2 n-2}-x_{2 n-1}\right\|^{2}+\left(\beta_{3}+\frac{\beta_{5}}{2}+\frac{\beta_{6}}{2}\right)\left\|x_{2 n-1}-x_{2 n}\right\|^{2} \\
& +\left(\beta_{4}+\frac{\beta_{5}}{2}+\frac{\beta_{6}}{2}\right)\left(\left\|x_{2 n-2}-x_{2 n-1}\right\|^{2}+\left\|x_{2 n}-x_{2 n-1}\right\|^{2}\right) \\
= & \left(\beta_{1}+\beta_{2}+\beta_{4}+\beta_{5}+\beta_{6}\right)\left\|x_{2 n-2}-x_{2 n-1}\right\|^{2}+\left(\beta_{3}\right. \\
& \left.+\beta_{4}+\beta_{5}+\beta_{6}\right)\left\|x_{2 n-1}-x_{2 n}\right\|^{2}
\end{aligned}
$$

(3.4) imply that

$$
\left\|x_{2 n-1}-x_{2 n}\right\|^{2} \leq \delta_{1}\left\|x_{2 n-2}-x_{2 n-1}\right\|^{2}
$$

where

$$
0<\delta_{1}=\frac{\beta_{1}+\beta_{2}+\beta_{4}+\beta_{5}+\beta_{6}}{1-\left(\beta_{3}+\beta_{4}+\beta_{5}+\beta_{6}\right)} .
$$

Again, from (3.1), parallelogram law, Lemma 2.3 and the fact that $(a, b)^{2} \leq a^{2}+b^{2}$, we obtain the following estimates:

$$
\begin{aligned}
& \left\|x_{2 n-2}-x_{2 n-1}\right\|^{2} \\
\leq & D_{1}^{2}\left(\Gamma_{1}\left(x_{2 n-3}\right), \Gamma_{2}\left(x_{2 n-2}\right)\right) \leq D^{2}\left(\Gamma_{1}\left(x_{2 n-3}\right), \Gamma_{2}\left(x_{2 n-2}\right)\right) \\
\leq \quad & \beta_{1}\left\|x_{2 n-3}-x_{2 n-2}\right\|^{2}+\beta_{2} P_{\alpha}^{2}\left(x_{2 n-3}, \Gamma_{1}\left(x_{2 n-3}\right)\right)+\beta_{3} P_{\alpha}^{2}\left(x_{2 n-2}, \Gamma_{2}\left(x_{2 n-2}\right)\right) \\
& +\beta_{4}\left\{\frac{P_{\alpha}^{2}\left(x_{2 n-2}, \Gamma_{1}\left(x_{2 n-3}\right)\right)+P_{\alpha}^{2}\left(x_{2 n-3}, \Gamma_{2}\left(x_{2 n-2}\right)\right)}{1+P_{\alpha}^{2}\left(x_{2 n-2}, \Gamma_{1}\left(x_{2 n-3}\right)\right) P_{\alpha}^{2}\left(x_{2 n-3}, \Gamma_{2}\left(x_{2 n-2}\right)\right)}\right\} \\
+ & \frac{\beta_{5}}{2}\left\{\left(P_{\alpha}^{2}\left(x_{2 n-3}, \Gamma_{1}\left(x_{2 n-3}\right)\right)+\frac{P_{\alpha}^{2}\left(x_{2 n-2}, \Gamma_{1}\left(x_{2 n-3}\right)\right)+P_{\alpha}^{2}\left(x_{2 n-3}, \Gamma_{2}\left(x_{2 n-2}\right)\right)}{1+P_{\alpha}^{2}\left(x_{2 n-2}, \Gamma_{1}\left(x_{2 n-3}\right)\right) P_{\alpha}^{2}\left(x_{2 n-3}, \Gamma_{2}\left(x_{2 n-2}\right)\right)}\right)\right. \\
+ & \left.\left(P_{\alpha}^{2}\left(x_{2 n-2}, \Gamma_{2}\left(x_{2 n-2}\right)\right)+\frac{P_{\alpha}^{2}\left(x_{2 n-3}, \Gamma_{1}\left(x_{2 n-3}\right)\right)+P_{\alpha}^{2}\left(x_{2 n-2}, \Gamma_{1}\left(x_{2 n-3}\right)\right)}{1+P_{\alpha}^{2}\left(x_{2 n-3}, \Gamma_{1}\left(x_{2 n-3}\right)\right) P_{\alpha}^{2}\left(x_{2 n-2}, \Gamma_{1}\left(x_{2 n-3}\right)\right)}\right)\right\} \\
+ & \frac{\beta_{6}}{2}\left\{\left(P_{\alpha}^{2}\left(x_{2 n-3}, \Gamma_{1}\left(x_{2 n-3}\right)\right)\right.\right. \\
+ & \left.\frac{P_{\alpha}^{2}\left(x_{2 n-3}, \Gamma_{2}\left(x_{2 n-2}\right)\right)+P_{\alpha}^{2}\left(x_{2 n-2}, \Gamma_{1}\left(x_{2 n-3}\right)\right) P_{\alpha}^{2}\left(x_{2 n-2}, \Gamma_{2}\left(x_{2 n-2}\right)\right)}{1+P_{\alpha}^{2}\left(x_{2 n-3}, \Gamma_{2}\left(x_{2 n-2}\right)\right) P_{\alpha}^{2}\left(x_{2 n-2}, \Gamma_{1}\left(x_{2 n-3}\right)\right) P_{\alpha}^{2}\left(x_{2 n-2}, \Gamma_{1}\left(x_{2 n-2}\right)\right)}\right) \\
+ & \left(P_{\alpha}^{2}\left(x_{2 n-3}, \Gamma_{2}\left(x_{2 n-2}\right)\right)\right.
\end{aligned}
$$




$$
\begin{aligned}
& \left.\left.+\frac{P_{\alpha}^{2}\left(x_{2 n-3}, \Gamma_{1}\left(x_{2 n-3}\right)\right)+P_{\alpha}^{2}\left(x_{2 n-2}, \Gamma_{2}\left(x_{2 n-3}\right)\right) P_{\alpha}^{2}\left(x_{2 n-2}, \Gamma_{2}\left(x_{2 n-2}\right)\right)}{1+P_{\alpha}^{2}\left(x_{2 n-3}, \Gamma_{1}\left(x_{2 n-3}\right)\right) P_{\alpha}^{2}\left(x_{2 n-2}, \Gamma_{2}\left(x_{2 n-3}\right)\right) P_{\alpha}^{2}\left(x_{2 n-2}, \Gamma_{2}\left(x_{2 n-2}\right)\right)}\right)\right\} \\
& \leq \beta_{1}\left\|x_{2 n-3}-x_{2 n-2}\right\|^{2}+\beta_{2}\left\|x_{2 n-3}-x_{2 n-2}\right\|^{2}+\beta_{3}\left\|x_{2 n-2}-x_{2 n-1}\right\|^{2} \\
& +\beta_{4}\left\{\frac{\left\|x_{2 n-2}-x_{2 n-2}\right\|^{2}+\left\|x_{2 n-3}-x_{2 n-1}\right\|^{2}}{1+\left\|x_{2 n-2}-x_{2 n-2}\right\|^{2}\left\|x_{2 n-3}-x_{2 n-1}\right\|^{2}}\right\} \\
& +\frac{\beta_{5}}{2}\left\{\left(\left\|x_{2 n-3}-x_{2 n-2}\right\|^{2}+\frac{\left\|x_{2 n-2}-x_{2 n-2}\right\|^{2}+\left\|x_{2 n-3}-x_{2 n-1}\right\|^{2}}{1+\left\|x_{2 n-2}-x_{2 n-2}\right\|^{2}\left\|x_{2 n-3}-x_{2 n-1}\right\|^{2}}\right)\right. \\
& \left.+\left(\left\|x_{2 n-2}-x_{2 n-1}\right\|^{2}+\frac{\left\|x_{2 n-3}-x_{2 n-2}\right\|^{2}+\left\|x_{2 n-2}-x_{2 n-2}\right\|^{2}}{1+\left\|x_{2 n-3}-x_{2 n-2}\right\|^{2}\left\|x_{2 n-2}-x_{2 n-2}\right\|^{2}}\right)\right\} \\
& +\frac{\beta_{6}}{2}\left\{\left(\left\|x_{2 n-3}-x_{2 n-2}\right\|^{2} x+\frac{\left\|x_{2 n-3}-x_{2 n-1}\right\|^{2}+\left\|x_{2 n-2}-x_{2 n-2}\right\|^{2}\left\|x_{2 n-2}-x_{2 n-1}\right\|^{2}}{1+\left\|x_{2 n-3}-x_{2 n-1}\right\|^{2}\left\|x_{2 n-2}-x_{2 n-2}\right\|^{2}\left\|x_{2 n-2}-x_{2 n-1}\right\|^{2}}\right)\right. \\
& \left.+\left(\left\|x_{2 n-3}-x_{2 n-1}\right\|^{2}+\frac{\left\|x_{2 n-3}-x_{2 n-2}\right\|^{2}+\left\|x_{2 n-2}-x_{2 n-2}\right\|^{2}\left\|x_{2 n-2}-x_{2 n-1}\right\|^{2}}{1+\left\|x_{2 n-3}-x_{2 n-2}\right\|^{2}\left\|x_{2 n-2}-x_{2 n-2}\right\|^{2}\left\|x_{2 n-2}-x_{2 n-1}\right\|^{2}}\right)\right\} \\
& =\beta_{1}\left\|x_{2 n-3}-x_{2 n-2}\right\|^{2}+\beta_{2}\left\|x_{2 n-3}-x_{2 n-2}\right\|^{2}+\beta_{3}\left\|x_{2 n-2}-x_{2 n-1}\right\|^{2}+\beta_{4}\left\|x_{2 n-3}-x_{2 n-1}\right\|^{2} \\
& +\frac{\beta_{5}}{2}\left(2\left\|x_{2 n-3}-x_{2 n-2}\right\|^{2}+2\left\|x_{2 n-3}-x_{2 n-1}\right\|^{2}\right)+\frac{\beta_{6}}{2}\left(2\left\|x_{2 n-3}-x_{2 n-2}\right\|^{2}+2\left\|x_{2 n-3}-x_{2 n-1}\right\|^{2}\right) \\
& =\beta_{1}\left\|x_{2 n-3}-x_{2 n-2}\right\|^{2}+\beta_{2}\left\|x_{2 n-3}-x_{2 n-2}\right\|^{2}+\beta_{3}\left\|x_{2 n-2}-x_{2 n-1}\right\|^{2}+\beta_{4}\left\|x_{2 n-3}-x_{2 n-1}\right\|^{2} \\
& +\frac{\beta_{5}}{2}\left(\left\|x_{2 n-3}-x_{2 n-2}+x_{2 n-3}-x_{2 n-1}\right\|^{2}+\left\|x_{2 n-3}-x_{2 n-2}-\left(x_{2 n-3}-x_{2 n-1}\right)\right\|^{2}\right) \\
& +\frac{\beta_{6}}{2}\left(\left\|x_{2 n-3}-x_{2 n-2}+x_{2 n-3}-x_{2 n-1}\right\|^{2}+\left\|x_{2 n-3}-x_{2 n-2}-\left(x_{2 n-3}-x_{2 n-1}\right)\right\|^{2}\right) \\
& =\beta_{1}\left\|x_{2 n-3}-x_{2 n-2}\right\|^{2}+\beta_{2}\left\|x_{2 n-3}-x_{2 n-2}\right\|^{2}+\beta_{3}\left\|x_{2 n-2}-x_{2 n-1}\right\|^{2}+\beta_{4}\left\|x_{2 n-3}-x_{2 n-1}\right\|^{2} \\
& +\frac{\beta_{5}}{2}\left(\left\|x_{2 n-3}-x_{2 n-2}-\left(x_{2 n-1}-x_{2 n-3}\right)\right\|^{2}+\left\|x_{2 n-1}-x_{2 n-3}\right\|^{2}\right) \\
& +\frac{\beta_{6}}{2}\left(\left\|x_{2 n-3}-x_{2 n-2}-\left(x_{2 n-1}-x_{2 n-3}\right)\right\|^{2}+\left\|x_{2 n-1}-x_{2 n-3}\right\|^{2}\right) \\
& \leq \beta_{1}\left\|x_{2 n-3}-x_{2 n-2}\right\|^{2}+\beta_{2}\left\|x_{2 n-3}-x_{2 n-2}\right\|^{2}+\beta_{3}\left\|x_{2 n-2}-x_{2 n-1}\right\|^{2}+\beta_{4}\left\|x_{2 n-3}-x_{2 n-1}\right\|^{2} \\
& +\frac{\beta_{5}}{2}\left(\left\|x_{2 n-3}-x_{2 n-2}\right\|^{2}+\left\|x_{2 n-1}-x_{2 n-3}\right\|^{2}+\left\|x_{2 n-1}-x_{2 n-3}\right\|^{2}\right) \\
& +\frac{\beta_{6}}{2}\left(\left\|x_{2 n-3}-x_{2 n-2}\right\|^{2}+\left\|x_{2 n-1}-x_{2 n-3}\right\|^{2}+\left\|x_{2 n-1}-x_{2 n-3}\right\|^{2}\right) \\
& =\left(\beta_{1}+\beta_{2}+\frac{\beta_{5}}{2}+\frac{\beta_{6}}{2}\right)\left\|x_{2 n-3}-x_{2 n-2}\right\|^{2}+\left(\beta_{3}+\frac{\beta_{5}}{2}+\frac{\beta_{6}}{2}\right)\left\|x_{2 n-2}-x_{2 n-1}\right\|^{2} \\
& +\left(\beta_{4}+\frac{\beta_{5}}{2}+\frac{\beta_{6}}{2}\right)\left\|x_{2 n-3}-x_{2 n-2}-\left(x_{2 n-1}-x_{2 n-2}\right)\right\|^{2} \\
& \leq\left(\beta_{1}+\beta_{2}+\frac{\beta_{5}}{2}+\frac{\beta_{6}}{2}\right)\left\|x_{2 n-3}-x_{2 n-2}\right\|^{2}+\left(\beta_{3}+\frac{\beta_{5}}{2}+\frac{\beta_{6}}{2}\right)\left\|x_{2 n-2}-x_{2 n-1}\right\|^{2} \\
& +\left(\beta_{4}+\frac{\beta_{5}}{2}+\frac{\beta_{6}}{2}\right)\left(\left\|x_{2 n-3}-x_{2 n-2}\right\|^{2}+\left\|x_{2 n-1}-x_{2 n-2}\right\|^{2}\right) \\
& (3.6)=\left(\beta_{1}+\beta_{2}+\beta_{4}+\beta_{5}+\beta_{6}\right)\left\|x_{2 n-3}-x_{2 n-2}\right\|^{2}+\left(\beta_{3}+\beta_{4}+\beta_{5}+\beta_{6}\right)\left\|x_{2 n-2}-x_{2 n-1}\right\|^{2}
\end{aligned}
$$

(3.6) imply that

$$
\left\|x_{2 n-2}-x_{2 n-1}\right\|^{2} \leq \delta_{2}\left\|x_{2 n-3}-x_{2 n-2}\right\|^{2}
$$

where

$$
0<\delta_{2}=\frac{\beta_{1}+\beta_{2}+\beta_{4}+\beta_{5}+\beta_{6}}{1-\left(\beta_{3}+\beta_{4}+\beta_{5}+\beta_{6}\right)} .
$$

Continuing in this manner as in above, we obtain (using (3.1), parallelogram law, Lemma 2.3 and the fact that $\left.(a, b)^{2} \leq a^{2}+b^{2}\right)$ that

$$
\left\|x_{1}-x_{2}\right\|^{2} \leq \delta_{n}\left\|x_{0}-x_{1}\right\|^{2},
$$


where

$$
0<\delta_{n}=\frac{\beta_{1}+\beta_{2}+\beta_{4}+\beta_{5}+\beta_{6}}{1-\left(\beta_{3}+\beta_{4}+\beta_{5}+\beta_{6}\right)} .
$$

Since $\delta_{1}=\delta_{2}=\delta_{3}=\cdots=\delta_{n}$, by letting $\delta=\delta_{1}$, we get (using (3.5)) that

$$
\left\|x_{2 n-1}-x_{2 n}\right\|^{2} \leq \delta^{n}\left\|x_{0}-x_{1}\right\|^{2} .
$$

In view of the fact that $\delta \in(0,1)$, we can conclude that $\left\{x_{n}\right\}$ is a Cauchy sequence in $H$ and hence converges to a point (say $\left.x^{\star}\right)$ in $H$.

Also, since by Lemma 2.3

$$
\begin{aligned}
& \left.P_{\alpha}^{2}\left(\Gamma_{1}\left(x^{\star}\right), x_{2 n}\right)\right) \\
& \leq D_{\alpha}^{2}\left(\Gamma_{1}\left(x^{\star}\right), \Gamma_{2}\left(x_{2 n-1}\right)\right) \leq D^{2}\left(\left(\Gamma_{1}\left(x^{\star}\right), \Gamma_{2}\left(x_{2 n-1}\right)\right)\right. \\
& \left.\leq \beta_{1}\left\|x^{\star}-x_{2 n-1}\right\|^{2}+\beta_{2} P_{\alpha}^{2}\left(x^{\star}, \Gamma_{1}\left(x^{\star}\right)\right)+\beta_{3} P_{\alpha}^{2}\left(x_{2 n-1}, \Gamma_{2} x_{2 n-1}\right)\right) \\
& +\beta_{4}\left\{\frac{P_{\alpha}^{2}\left(x_{2 n-1}, \Gamma_{1}\left(x^{\star}\right)\right)+P_{\alpha}^{2}\left(x^{\star}, \Gamma_{2}\left(x_{2 n-1}\right)\right)}{1+P_{\alpha}^{2}\left(x_{2 n-1}, \Gamma_{1}\left(x^{\star}\right)\right) P_{\alpha}^{2}\left(x^{\star}, \Gamma_{2}\left(x_{2 n-1}\right)\right)}\right\} \\
& +\frac{\beta_{5}}{2}\left\{\left(P_{\alpha}^{2}\left(x^{\star}, \Gamma_{1}\left(x^{\star}\right)\right)+\frac{P_{\alpha}^{2}\left(x_{2 n-1}, \Gamma_{1}\left(x^{\star}\right)\right)+P_{\alpha}^{2}\left(x^{\star}, \Gamma_{2}\left(x_{2 n-1}\right)\right)}{1+P_{\alpha}^{2}\left(x_{2 n-1}, \Gamma_{1}\left(x^{\star}\right)\right) P_{\alpha}^{2}\left(x^{\star}, \Gamma_{2}\left(x_{2 n-1}\right)\right)}\right)\right. \\
& \left.+\left(P_{\alpha}^{2}\left(x_{2 n-1}, \Gamma_{2}\left(x_{2 n-1}\right)\right)+\frac{P_{\alpha}^{2}\left(x^{\star}, \Gamma_{1}\left(x^{\star}\right)\right)+P_{\alpha}^{2}\left(x_{2 n-1}, \Gamma_{1}\left(x^{\star}\right)\right)}{1+P_{\alpha}^{2}\left(x^{\star}, \Gamma_{1}\left(x^{\star}\right)\right) P_{\alpha}^{2}\left(x_{2 n-1}, \Gamma_{1}\left(x^{\star}\right)\right)}\right)\right\} \\
& +\frac{\beta_{6}}{2}\left\{\left(P_{\alpha}^{2}\left(x^{\star}, \Gamma_{1}\left(x^{\star}\right)\right)\right.\right. \\
& \left.+\frac{P_{\alpha}^{2}\left(x^{\star}, \Gamma_{2}\left(x^{\star}\right)\right)+P_{\alpha}^{2}\left(x_{2 n-1}, \Gamma_{1}\left(x^{\star}\right)\right) P_{\alpha}^{2}\left(x_{2 n-1}, \Gamma_{2}\left(x_{2 n-1}\right)\right)}{1+P_{\alpha}^{2}\left(x^{\star}, \Gamma_{2}\left(x_{2 n-1}\right)\right) P_{\alpha}^{2}\left(x_{2 n-1}, \Gamma_{1}\left(x^{\star}\right)\right) P_{\alpha}^{2}\left(x_{2 n-1}, \Gamma_{1}\left(x_{2 n-1}\right)\right)}\right) \\
& +\left(P _ { \alpha } ^ { 2 } \left(x^{\star}, \Gamma_{2}\left(x_{2 n-1}\right)\right.\right. \\
& \left.\left.+\frac{P_{\alpha}^{2}\left(x^{\star}, \Gamma_{1}\left(x^{\star}\right)\right)+P_{\alpha}^{2}\left(x_{2 n-1}, \Gamma_{2}\left(x^{\star}\right)\right) P_{\alpha}^{2}\left(x_{2 n-1}, \Gamma_{2}\left(x_{2 n-1}\right)\right)}{1+P_{\alpha}^{2}\left(x^{\star}, \Gamma_{1}\left(x^{\star}\right)\right) P_{\alpha}^{2}\left(x_{2 n-1}, \Gamma_{2}\left(x^{\star}\right)\right) P_{\alpha}^{2}\left(x_{2 n-1}, \Gamma_{2}\left(x_{2 n-1}\right)\right)}\right)\right\} \\
& \left.\leq \beta_{1}\left\|x^{\star}-x_{2 n-1}\right\|^{2}+\beta_{2} P_{\alpha}^{2}\left(x^{\star}, \Gamma_{1}\left(x^{\star}\right)\right)+\beta_{3} P_{\alpha}^{2}\left(x_{2 n-1}, x_{2 n}\right)\right) \\
& +\beta_{4}\left\{\frac{P_{\alpha}^{2}\left(x_{2 n-1}, \Gamma_{1}\left(x^{\star}\right)\right)+P_{\alpha}^{2}\left(x^{\star}, x_{2 n}\right)}{1+P_{\alpha}^{2}\left(x_{2 n-1}, \Gamma\left(x^{\star}\right)\right) P_{\alpha}^{2}\left(x^{\star}, x_{2 n}\right)}\right\} \\
& +\frac{\beta_{5}}{2}\left\{\left(P_{\alpha}^{2}\left(x^{\star}, \Gamma_{1}\left(x^{\star}\right)\right)+\frac{P_{\alpha}^{2}\left(x_{2 n-1}, \Gamma_{1}\left(x^{\star}\right)\right)+P_{\alpha}^{2}\left(x^{\star},\left(x_{2 n}\right)\right.}{1+P_{\alpha}^{2}\left(x_{2 n-1}, \Gamma_{1}\left(x^{\star}\right)\right) P_{\alpha}^{2}\left(x^{\star}, x_{2 n}\right)}\right)\right. \\
& \left.+\left(P_{\alpha}^{2}\left(x_{2 n-1}, x_{2 n}\right)+\frac{P_{\alpha}^{2}\left(x^{\star}, \Gamma_{1}\left(x^{\star}\right)\right)+P_{\alpha}^{2}\left(x_{2 n-1}, \Gamma_{1}\left(x^{\star}\right)\right)}{1+P_{\alpha}^{2}\left(x^{\star}, \Gamma_{1}\left(x^{\star}\right)\right) P_{\alpha}^{2}\left(x_{2 n-1}, \Gamma_{1}\left(x^{\star}\right)\right)}\right)\right\} \\
& +\frac{\beta_{6}}{2}\left\{\left(P_{\alpha}^{2}\left(x^{\star}, \Gamma_{1}\left(x^{\star}\right)\right)+\frac{P_{\alpha}^{2}\left(x^{\star}, x_{2 n}\right)+P_{\alpha}^{2}\left(x_{2 n-1}, \Gamma_{1}\left(x^{\star}\right)\right) P_{\alpha}^{2}\left(x_{2 n-1}, x_{2 n}\right)}{1+P_{\alpha}^{2}\left(x_{2 n-2}, x_{2 n}\right) P_{\alpha}^{2}\left(x_{2 n-1}, \Gamma_{1}\left(x^{\star}\right)\right) P_{\alpha}^{2}\left(x_{2 n-1}, x_{2 n}\right)}\right)\right. \\
& \left.+\left(P_{\alpha}^{2}\left(x^{\star}, x_{2 n}\right)+\frac{P_{\alpha}^{2}\left(x^{\star}, \Gamma_{1}\left(x^{\star}\right)\right)+P_{\alpha}^{2}\left(x_{2 n-1}, \Gamma_{1}\left(x^{\star}\right)\right) P_{\alpha}^{2}\left(x_{2 n-1}, x_{2 n}\right)}{1+P_{\alpha}^{2}\left(x^{\star}, \Gamma_{1}\left(x^{\star}\right)\right) P_{\alpha}^{2}\left(x_{2 n-1}, \Gamma_{1}\left(x^{\star}\right)\right) P_{\alpha}^{2}\left(x_{2 n-1}, x_{2 n}\right)}\right)\right\},
\end{aligned}
$$


it follows (from the continuity of $P_{\alpha}$ ) that

$$
\left.P_{\alpha}^{2}\left(\Gamma_{1}\left(x^{\star}\right), x^{\star}\right)\right) \leq\left(\beta_{2}+\beta_{4}+2 \beta_{5}+\beta_{6}\right) P_{\alpha}^{2}\left(x^{\star}, \Gamma_{1}\left(x^{\star}\right)\right)
$$

as $n \rightarrow \infty$. Since $\beta_{2}+\beta_{4}+2 \beta_{5}+\beta_{6}<1$, it follows that $\left.P_{\alpha}^{2}\left(\Gamma_{1}\left(x^{\star}\right), x^{\star}\right)\right)=0$. Thus, by Lemma 2.5, we have $x^{\star} \subset \Gamma_{1} x^{\star}$. Using the same argument as above, we get $x^{\star} \subset \Gamma_{2} x^{\star}$. Consequently, $x^{\star} \subset \Gamma_{1} x^{\star} \cap \Gamma_{2} x^{\star}$. This completes the proof of Theorem 3.1

Theorem 3.2. Let $H$ be a Hilbert space and let $\Gamma_{1}, \Gamma_{2}: H \longrightarrow \mathcal{W}(H)$ a pair of fuzzy mappings satisfying

$$
\begin{aligned}
D^{2}\left(\Gamma_{1} x, \Gamma_{2} y\right) \leq & q \max \left\{\|x-y\|^{2}, P_{\alpha}^{2}\left(x, \Gamma_{1} x\right), P_{\alpha}^{2}\left(y, \Gamma_{2} y\right),\left\{\frac{P_{\alpha}^{2}\left(y, \Gamma_{1} x\right)+P_{\alpha}^{2}\left(x, \Gamma_{2} y\right)}{1+P_{\alpha}^{2}\left(y, \Gamma_{1} x\right) P_{\alpha}^{2}\left(x, \Gamma_{2} y\right)}\right\},\right. \\
& \frac{1}{2}\left\{\left(P_{\alpha}^{2}\left(x, \Gamma_{1} x\right)+\frac{P_{\alpha}^{2}\left(y, \Gamma_{1} x\right)+P_{\alpha}^{2}\left(x, \Gamma_{2} y\right)}{1+P_{\alpha}^{2}\left(y, \Gamma_{1} x\right) P_{\alpha}^{2}\left(x, \Gamma_{2} y\right)}\right)\right. \\
& \left.+\left(P_{\alpha}^{2}\left(y, \Gamma_{1} x\right)+\frac{P_{\alpha}^{2}\left(x, \Gamma_{1} x\right)+P_{\alpha}^{2}\left(x, \Gamma_{2} y\right)}{1+P_{\alpha}^{2}\left(x, \Gamma_{1} x\right) P_{\alpha}^{2}\left(x, \Gamma_{2} y\right)}\right)\right\}, \\
& \frac{1}{2}\left\{\left(P_{\alpha}^{2}\left(x, \Gamma_{1} x\right)+\frac{P_{\alpha}^{2}\left(y, \Gamma_{1} x\right)+P_{\alpha}^{2}\left(x, \Gamma_{2} y\right) P_{\alpha}^{2}\left(y, \Gamma_{2} y\right)}{1+P_{\alpha}^{2}\left(y, \Gamma_{1} x\right) P_{\alpha}^{2}\left(x, \Gamma_{2} y\right) P_{\alpha}^{2}\left(y, \Gamma_{1} y\right)}\right)\right. \\
& \left.\left.+\left(P_{\alpha}^{2}\left(y, \Gamma_{1} x\right)+\frac{P_{\alpha}^{2}\left(x, \Gamma_{1} x\right)+P_{\alpha}^{2}\left(x, \Gamma_{2} y\right) P_{\alpha}^{2}\left(y, \Gamma_{2} y\right)}{1+P_{\alpha}^{2}\left(x, \Gamma_{1} x\right) P_{\alpha}^{2}\left(x, \Gamma_{2} y\right) P_{\alpha}^{2}\left(y, \Gamma_{2} y\right)}\right)\right\}\right\}
\end{aligned}
$$

for all $x, y \in H$ and for all $\alpha \in[0,1]$, where $q \in\left(0, \frac{1}{4}\right)$. Then, there exists a point $x^{\star} \in H$ such that $\left\{x^{\star}\right\} \in \Gamma_{1} x^{\star} \cap \Gamma_{2} x^{\star}$.

Proof. Let $x_{0} \in H$ and the sequence $\left\{x_{n}\right\}$ be as in Theorem 3.1. Then, using (3.1), Lemma 2.3 and the fact that $(a-b)^{2} \leq a^{2}+b^{2}$, we estimate as follows:

$$
\begin{aligned}
& \left\|x_{2 n-1}-x_{2 n}\right\|^{2} \\
\leq & D_{1}^{2}\left(\Gamma_{1}\left(x_{2 n-2}\right), \Gamma_{2}\left(x_{2 n-1}\right)\right) \leq D^{2}\left(\Gamma_{1}\left(x_{2 n-2}\right), \Gamma_{2}\left(x_{2 n-1}\right)\right) \\
\leq & q \max \left\{\left\|x_{2 n-2}-x_{2 n-1}\right\|^{2}, P_{\alpha}^{2}\left(x_{2 n-2}, \Gamma_{1}\left(x_{2 n-2}\right)\right), P_{\alpha}^{2}\left(x_{2 n-1}, \Gamma_{2}\left(x_{2 n-1}\right)\right),\right. \\
& \left\{\frac{P_{\alpha}^{2}\left(x_{2 n-1}, \Gamma_{1}\left(x_{2 n-2}\right)\right)+P_{\alpha}^{2}\left(x_{2 n-2}, \Gamma_{2}\left(x_{2 n-1}\right)\right)}{1+P_{\alpha}^{2}\left(x_{2 n-1}, \Gamma_{1}\left(x_{2 n-2}\right)\right) P_{\alpha}^{2}\left(x_{2 n-2}, \Gamma_{2}\left(x_{2 n-1}\right)\right)}\right\}, \\
& \frac{1}{2}\left\{\left(P_{\alpha}^{2}\left(x_{2 n-2}, \Gamma_{1}\left(x_{2 n-2}\right)\right)+\frac{P_{\alpha}^{2}\left(x_{2 n-1}, \Gamma_{1}\left(x_{2 n-2}\right)\right)+P_{\alpha}^{2}\left(x_{2 n-2}, \Gamma_{2}\left(x_{2 n-1}\right)\right)}{1+P_{\alpha}^{2}\left(x_{2 n-1}, \Gamma_{1}\left(x_{2 n-2}\right)\right) P_{\alpha}^{2}\left(x_{2 n-2}, \Gamma_{2}\left(x_{2 n-1}\right)\right)}\right)\right.
\end{aligned}
$$


$\left.+\left(P_{\alpha}^{2}\left(x_{2 n-1}, \Gamma_{2}\left(x_{2 n-1}\right)\right)+\frac{P_{\alpha}^{2}\left(x_{2 n-2}, \Gamma_{1}\left(x_{2 n-2}\right)\right)+P_{\alpha}^{2}\left(x_{2 n-1}, \Gamma_{1}\left(x_{2 n-2}\right)\right)}{1+P_{\alpha}^{2}\left(x_{2 n-2}, \Gamma_{1}\left(x_{2 n-2}\right)\right) P_{\alpha}^{2}\left(x_{2 n-1}, \Gamma_{1}\left(x_{2 n-2}\right)\right)}\right)\right\}$,

$\frac{1}{2}\left\{\left(P_{\alpha}^{2}\left(x_{2 n-2}, \Gamma_{1}\left(x_{2 n-2}\right)\right)\right.\right.$

$\left.+\frac{P_{\alpha}^{2}\left(x_{2 n-2}, \Gamma_{2}\left(x_{2 n-1}\right)\right)+P_{\alpha}^{2}\left(x_{2 n-1}, \Gamma_{1}\left(x_{2 n-2}\right)\right) P_{\alpha}^{2}\left(x_{2 n-1}, \Gamma_{2}\left(x_{2 n-1}\right)\right)}{1+P_{\alpha}^{2}\left(x_{2 n-2}, \Gamma_{2}\left(x_{2 n-1}\right)\right) P_{\alpha}^{2}\left(x_{2 n-1}, \Gamma_{1}\left(x_{2 n-2}\right)\right) P_{\alpha}^{2}\left(x_{2 n-1}, \Gamma_{1}\left(x_{2 n-1}\right)\right)}\right)$

$+\left(P_{\alpha}^{2}\left(x_{2 n-2}, \Gamma_{2}\left(x_{2 n-1}\right)\right)\right.$

$\left.\left.\left.+\frac{P_{\alpha}^{2}\left(x_{2 n-2}, \Gamma_{1}\left(x_{2 n-2}\right)\right)+P_{\alpha}^{2}\left(x_{2 n-1}, \Gamma_{2}\left(x_{2 n-2}\right)\right) P_{\alpha}^{2}\left(x_{2 n-1}, \Gamma_{2}\left(x_{2 n-1}\right)\right)}{1+P_{\alpha}^{2}\left(x_{2 n-2}, \Gamma_{1}\left(x_{2 n-2}\right)\right) P_{\alpha}^{2}\left(x_{2 n-1}, \Gamma_{2}\left(x_{2 n-2}\right)\right) P_{\alpha}^{2}\left(x_{2 n-1}, \Gamma_{2}\left(x_{2 n-1}\right)\right)}\right)\right\}\right\}$

$\leq \operatorname{qmax}\left\{\left\|x_{2 n-2}-x_{2 n-1}\right\|^{2},\left\|x_{2 n-2}-x_{2 n-1}\right\|^{2},\left\|x_{2 n-1}-x_{2 n}\right\|^{2}\right.$,

$\left\{\frac{\left\|x_{2 n-1}-x_{2 n-1}\right\|^{2}+\left\|x_{2 n-2}-x_{2 n}\right\|^{2}}{1+\left\|x_{2 n-1}-x_{2 n-1}\right\|^{2}\left\|x_{2 n-2}-x_{2 n}\right\|^{2}}\right\}$,

$\frac{1}{2}\left\{\left(\left\|x_{2 n-2}-x_{2 n-2}\right\|^{2}+\frac{\left\|x_{2 n-1}-x_{2 n-1}\right\|^{2}+\left\|x_{2 n-2}-x_{2 n}\right\|^{2}}{1+\left\|x_{2 n-1}-x_{2 n-1}\right\|^{2}\left\|x_{2 n-2}-x_{2 n-1}\right\|^{2}}\right)\right.$

$\left.+\left(\left\|x_{2 n-1}-x_{2 n}\right\|^{2}+\frac{\left\|x_{2 n-2}-x_{2 n-1}\right\|^{2}+\left\|x_{2 n-1}-x_{2 n-1}\right\|^{2}}{1+\left\|x_{2 n-2}-x_{2 n-1}\right\|^{2}\left\|x_{2 n-1}-x_{2 n-1}\right\|^{2}}\right)\right\}$,

$\frac{1}{2}\left\{\left(\left\|x_{2 n-2}-x_{2 n-1}\right\|^{2} x+\frac{\left\|x_{2 n-2}-x_{2 n}\right\|^{2}+\left\|x_{2 n-1}-x_{2 n-1}\right\|^{2}\left\|x_{2 n-1}-x_{2 n}\right\|^{2}}{1+\left\|x_{2 n-2}-x_{2 n}\right\|^{2}\left\|x_{2 n-1}-x_{2 n-1}\right\|^{2}\left\|x_{2 n-1}-x_{2 n}\right\|^{2}}\right)\right.$

$\left.\left.+\left(\left\|x_{2 n-2}-x_{2 n}\right\|^{2}+\frac{\left\|x_{2 n-2}-x_{2 n-1}\right\|^{2}+\left\|x_{2 n-1}-x_{2 n-1}\right\|^{2}\left\|2 n-1-x_{2 n}\right\|^{2}}{1+\left\|x_{2 n-2}-x_{2 n-1}\right\|^{2}\left\|x_{2 n-1}-x_{2 n-1}\right\|^{2}\left\|x_{2 n-1}-x_{2 n}\right\|^{2}}\right)\right\}\right\}$

$=\operatorname{qmax}\left\{\left\|x_{2 n-2}-x_{2 n-1}\right\|^{2},\left\|x_{2 n-1}-x_{2 n}\right\|^{2},\left\|x_{2 n-2}-x_{2 n}\right\|^{2}\right.$,

$\left.\frac{1}{2}\left(2\left\|x_{2 n-2}-x_{2 n-1}\right\|^{2}+2\left\|x_{2 n-2}-x_{2 n}\right\|^{2}\right)\right\}$

$=q \max \left\{\left\|x_{2 n-2}-x_{2 n-1}\right\|^{2},\left\|x_{2 n-1}-x_{2 n}\right\|^{2},\left\|x_{2 n-2}-x_{2 n}\right\|^{2}\right.$,

$\left.\frac{1}{2}\left(2\left\|x_{2 n-2}-x_{2 n-1}\right\|^{2}+2\left\|x_{2 n-2}-x_{2 n-1}-\left(x_{2 n}-x_{2 n-1}\right)\right\|^{2}\right)\right\}$

$\leq q \operatorname{qax}\left\{\left\|x_{2 n-2}-x_{2 n-1}\right\|^{2},\left\|x_{2 n-1}-x_{2 n}\right\|^{2},\left\|x_{2 n-2}-x_{2 n}\right\|^{2}, 2\left\|x_{2 n-2}-x_{2 n-1}\right\|^{2}\right.$

$\left.\left.+\left\|x_{2 n}-x_{2 n-1}\right\|^{2}\right)\right\}$

$\left.(3.11)=q\left(2\left\|x_{2 n-2}-x_{2 n-1}\right\|^{2}+\left\|x_{2 n}-x_{2 n-1}\right\|^{2}\right)\right)$ 
(3.11) implies

$$
\left\|x_{2 n-1}-x_{2 n}\right\|^{2} \leq \gamma_{1}\left\|x_{2 n-2}-x_{2 n-1}\right\|^{2}
$$

where $0<\gamma_{1}=\frac{2 q}{1-q}<1$.

Using (3.1), Lemma 2.3 and the fact that $(a, b)^{2} \leq a^{2}+b^{2}$, we get the following estimate for $\left\|x_{2 n-2}-x_{2 n-1}\right\|^{2}$ :

$$
\begin{aligned}
& \left\|x_{2 n-2}-x_{2 n-1}\right\|^{2} \\
& \leq D_{1}^{2}\left(\Gamma_{1}\left(x_{2 n-3}\right), \Gamma_{2}\left(x_{2 n-2}\right)\right) \leq D^{2}\left(\Gamma_{1}\left(x_{2 n-3}\right), \Gamma_{2}\left(x_{2 n-2}\right)\right) \\
& \leq q \operatorname{qmax}\left\{\left\|x_{2 n-3}-x_{2 n-2}\right\|^{2}, P_{\alpha}^{2}\left(x_{2 n-3}, \Gamma_{1}\left(x_{2 n-3}\right)\right), P_{\alpha}^{2}\left(x_{2 n-2}, \Gamma_{2}\left(x_{2 n-2}\right)\right)\right. \text {, } \\
& \left\{\frac{P_{\alpha}^{2}\left(x_{2 n-2}, \Gamma_{1}\left(x_{2 n-3}\right)\right)+P_{\alpha}^{2}\left(x_{2 n-3}, \Gamma_{2}\left(x_{2 n-2}\right)\right)}{1+P_{\alpha}^{2}\left(x_{2 n-2}, \Gamma_{1}\left(x_{2 n-3}\right)\right) P_{\alpha}^{2}\left(x_{2 n-3}, \Gamma_{2}\left(x_{2 n-2}\right)\right)}\right\}, \\
& \frac{1}{2}\left\{\left(P_{\alpha}^{2}\left(x_{2 n-3}, \Gamma_{1}\left(x_{2 n-3}\right)\right)+\frac{P_{\alpha}^{2}\left(x_{2 n-2}, \Gamma_{1}\left(x_{2 n-3}\right)\right)+P_{\alpha}^{2}\left(x_{2 n-3}, \Gamma_{2}\left(x_{2 n-2}\right)\right)}{1+P_{\alpha}^{2}\left(x_{2 n-2}, \Gamma_{1}\left(x_{2 n-3}\right)\right) P_{\alpha}^{2}\left(x_{2 n-3}, \Gamma_{2}\left(x_{2 n-2}\right)\right)}\right)\right. \\
& \left.+\left(P_{\alpha}^{2}\left(x_{2 n-2}, \Gamma_{2}\left(x_{2 n-2}\right)\right)+\frac{P_{\alpha}^{2}\left(x_{2 n-3}, \Gamma_{1}\left(x_{2 n-3}\right)\right)+P_{\alpha}^{2}\left(x_{2 n-2}, \Gamma_{1}\left(x_{2 n-3}\right)\right)}{1+P_{\alpha}^{2}\left(x_{2 n-3}, \Gamma_{1}\left(x_{2 n-3}\right)\right) P_{\alpha}^{2}\left(x_{2 n-2}, \Gamma_{1}\left(x_{2 n-3}\right)\right)}\right)\right\}, \\
& \frac{1}{2}\left\{\left(P_{\alpha}^{2}\left(x_{2 n-3}, \Gamma_{1}\left(x_{2 n-3}\right)\right)\right.\right. \\
& \left.+\frac{P_{\alpha}^{2}\left(x_{2 n-3}, \Gamma_{2}\left(x_{2 n-2}\right)\right)+P_{\alpha}^{2}\left(x_{2 n-2}, \Gamma_{1}\left(x_{2 n-3}\right)\right) P_{\alpha}^{2}\left(x_{2 n-2}, \Gamma_{2}\left(x_{2 n-2}\right)\right)}{1+P_{\alpha}^{2}\left(x_{2 n-3}, \Gamma_{2}\left(x_{2 n-2}\right)\right) P_{\alpha}^{2}\left(x_{2 n-2}, \Gamma_{1}\left(x_{2 n-3}\right)\right) P_{\alpha}^{2}\left(x_{2 n-2}, \Gamma_{1}\left(x_{2 n-2}\right)\right)}\right) \\
& +\left(P_{\alpha}^{2}\left(x_{2 n-3}, \Gamma_{2}\left(x_{2 n-2}\right)\right)\right. \\
& \left.\left.\left.+\frac{P_{\alpha}^{2}\left(x_{2 n-3}, \Gamma_{1}\left(x_{2 n-3}\right)\right)+P_{\alpha}^{2}\left(x_{2 n-2}, \Gamma_{2}\left(x_{2 n-3}\right)\right) P_{\alpha}^{2}\left(x_{2 n-2}, \Gamma_{2}\left(x_{2 n-2}\right)\right)}{1+P_{\alpha}^{2}\left(x_{2 n-3}, \Gamma_{1}\left(x_{2 n-3}\right)\right) P_{\alpha}^{2}\left(x_{2 n-2}, \Gamma_{2}\left(x_{2 n-3}\right)\right) P_{\alpha}^{2}\left(x_{2 n-2}, \Gamma_{2}\left(x_{2 n-2}\right)\right)}\right)\right\}\right\} \\
& \leq q \operatorname{qax}\left\{\left\|x_{2 n-3}-x_{2 n-2}\right\|^{2},\left\|x_{2 n-3}-x_{2 n-2}\right\|^{2}+\beta_{3}\left\|x_{2 n-2}-x_{2 n-1}\right\|^{2},\right. \\
& \left\{\frac{\left\|x_{2 n-2}-x_{2 n-2}\right\|^{2}+\left\|x_{2 n-3}-x_{2 n-1}\right\|^{2}}{1+\left\|x_{2 n-2}-x_{2 n-2}\right\|^{2}\left\|x_{2 n-3}-x_{2 n-1}\right\|^{2}}\right\} \text {, } \\
& \frac{1}{2}\left\{\left(\left\|x_{2 n-3}-x_{2 n-2}\right\|^{2}+\frac{\left\|x_{2 n-2}-x_{2 n-2}\right\|^{2}+\left\|x_{2 n-3}-x_{2 n-1}\right\|^{2}}{1+\left\|x_{2 n-2}-x_{2 n-2}\right\|^{2}\left\|x_{2 n-3}-x_{2 n-1}\right\|^{2}}\right)\right. \\
& \left.+\left(\left\|x_{2 n-2}-x_{2 n-1}\right\|^{2}+\frac{\left\|x_{2 n-3}-x_{2 n-2}\right\|^{2}+\left\|x_{2 n-2}-x_{2 n-2}\right\|^{2}}{1+\left\|x_{2 n-3}-x_{2 n-2}\right\|^{2}\left\|x_{2 n-2}-x_{2 n-2}\right\|^{2}}\right)\right\}, \\
& \frac{1}{2}\left\{\left(\left\|x_{2 n-3}-x_{2 n-2}\right\|^{2} x\right.\right.
\end{aligned}
$$




$$
\begin{aligned}
& \left.+\frac{\left\|x_{2 n-3}-x_{2 n-1}\right\|^{2}+\left\|x_{2 n-2}-x_{2 n-2}\right\|^{2}\left\|x_{2 n-2}-x_{2 n-1}\right\|^{2}}{\left.\left.1+\left\|x_{2 n-3}-x_{2 n-1}\right\|^{2}\left\|x_{2 n-2}-x_{2 n-2}\right\|^{2} \| x_{2 n-2}-x_{2 n-1}\right)\right)}\right) \\
& +\left(\left\|x_{2 n-3}-x_{2 n-1}\right\|^{2}\right. \\
& \left.\left.\left.+\frac{\left\|x_{2 n-3}-x_{2 n-2}\right\|^{2}+\left\|x_{2 n-2}-x_{2 n-2}\right\|^{2}\left\|x_{2 n-2}-x_{2 n-1}\right\|^{2}}{1+\left\|x_{2 n-3}-x_{2 n-2}\right\|^{2}\left\|x_{2 n-2}-x_{2 n-2}\right\|^{2}\left\|x_{2 n-2}-x_{2 n-1}\right\|^{2}}\right)\right\}\right\} \\
& =\quad q m a x\left\{\left\|x_{2 n-3}-x_{2 n-2}\right\|^{2},\left\|x_{2 n-2}-x_{2 n-1}\right\|^{2},\left\|x_{2 n-3}-x_{2 n-1}\right\|^{2},\right. \\
& \left.\quad \frac{1}{2}\left(2\left\|x_{2 n-3}-x_{2 n-2}\right\|^{2}+2\left\|x_{2 n-3}-x_{2 n-2}-\left(x_{2 n-1}-x_{2 n-2}\right)\right\|^{2}\right)\right\} \\
& \leq \quad q m a x\left\{\left\|x_{2 n-3}-x_{2 n-2}\right\|^{2},\left\|x_{2 n-2}-x_{2 n-1}\right\|^{2},\left\|x_{2 n-3}-x_{2 n-1}\right\|^{2},\right. \\
& \left.\quad\left(2\left\|x_{2 n-3}-x_{2 n-2}\right\|^{2}+\left\|x_{2 n-1}-x_{2 n-2}\right\|^{2}\right)\right\} \\
& =2 q\left\|x_{2 n-3}-x_{2 n-2}\right\|^{2}+q\left\|x_{2 n-1}-x_{2 n-2}\right\|^{2}
\end{aligned}
$$

(3.13) implies

$$
\left\|x_{2 n-2}-x_{2 n-1}\right\|^{2} \leq \gamma_{2}\left\|x_{2 n-3}-x_{2 n-2}\right\|^{2}
$$

where $0<\gamma_{2}=\frac{2 q}{1-q}<1$.

By continuing in this process, using the same argument as in the above, we will get

$$
\left\|x_{2 n-1}-x_{2 n}\right\|^{2} \leq \gamma_{1} \gamma_{2} \cdots \gamma_{n}\left\|x_{0}-x_{1}\right\|^{2}
$$

Since $\gamma_{1}=\gamma_{2}=\gamma_{3}=\cdots=\gamma_{n}, n \in \mathbb{N}$, it follows from (3.15) (with $\gamma_{1}=\gamma$ ) that

$$
\left\|x_{2 n-1}-x_{2 n}\right\|^{2} \leq \gamma^{n}\left\|x_{0}-x_{1}\right\|^{2}
$$

Using the fact that $\gamma \in(0,1)$, we can conclude (from (3.16)) that $\left\{x_{n}\right\}$ is a Cauchy sequence in $H$ and hence converges to a point (say $x^{\star}$ ) in $H$. 
Now, since by Lemma 2.3

$$
\begin{aligned}
& \left.P_{\alpha}^{2}\left(\Gamma_{1}\left(x^{\star}\right), x_{2 n}\right)\right) \\
& \leq D_{\alpha}^{2}\left(\Gamma_{1}\left(x^{\star}\right), \Gamma_{2}\left(x_{2 n-1}\right)\right) \leq D^{2}\left(\left(\Gamma_{1}\left(x^{\star}\right), \Gamma_{2}\left(x_{2 n-1}\right)\right)\right. \\
& \leq \operatorname{qmax}\left\{\left\|x^{\star}-x_{2 n-1}\right\|^{2}, P_{\alpha}^{2}\left(x^{\star}, \Gamma_{1}\left(x^{\star}\right)\right), P_{\alpha}^{2}\left(x_{2 n-1}, \Gamma_{2} x_{2 n-1}\right)\right), \\
& \left\{\frac{P_{\alpha}^{2}\left(x_{2 n-1}, \Gamma_{1}\left(x^{\star}\right)\right)+P_{\alpha}^{2}\left(x^{\star}, \Gamma_{2}\left(x_{2 n-1}\right)\right)}{1+P_{\alpha}^{2}\left(x_{2 n-1}, \Gamma_{1}\left(x^{\star}\right)\right) P_{\alpha}^{2}\left(x^{\star}, \Gamma_{2}\left(x_{2 n-1}\right)\right)}\right\}, \\
& \frac{1}{2}\left\{\left(P_{\alpha}^{2}\left(x^{\star}, \Gamma_{1}\left(x^{\star}\right)\right)+\frac{P_{\alpha}^{2}\left(x_{2 n-1}, \Gamma_{1}\left(x^{\star}\right)\right)+P_{\alpha}^{2}\left(x^{\star}, \Gamma_{2}\left(x_{2 n-1}\right)\right)}{1+P_{\alpha}^{2}\left(x_{2 n-1}, \Gamma_{1}\left(x^{\star}\right)\right) P_{\alpha}^{2}\left(x^{\star}, \Gamma_{2}\left(x_{2 n-1}\right)\right)}\right)\right. \\
& \left.+\left(P_{\alpha}^{2}\left(x_{2 n-1}, \Gamma_{2}\left(x_{2 n-1}\right)\right)+\frac{P_{\alpha}^{2}\left(x^{\star}, \Gamma_{1}\left(x^{\star}\right)\right)+P_{\alpha}^{2}\left(x_{2 n-1}, \Gamma_{1}\left(x^{\star}\right)\right)}{1+P_{\alpha}^{2}\left(x^{\star}, \Gamma_{1}\left(x^{\star}\right)\right) P_{\alpha}^{2}\left(x_{2 n-1}, \Gamma_{1}\left(x^{\star}\right)\right)}\right)\right\}, \\
& \frac{1}{2}\left\{\left(P_{\alpha}^{2}\left(x^{\star}, \Gamma_{1}\left(x^{\star}\right)\right)\right.\right. \\
& \left.+\frac{P_{\alpha}^{2}\left(x^{\star}, \Gamma_{2}\left(x^{\star}\right)\right)+P_{\alpha}^{2}\left(x_{2 n-1}, \Gamma_{1}\left(x^{\star}\right)\right) P_{\alpha}^{2}\left(x_{2 n-1}, \Gamma_{2}\left(x_{2 n-1}\right)\right)}{1+P_{\alpha}^{2}\left(x^{\star}, \Gamma_{2}\left(x_{2 n-1}\right)\right) P_{\alpha}^{2}\left(x_{2 n-1}, \Gamma_{1}\left(x^{\star}\right)\right) P_{\alpha}^{2}\left(x_{2 n-1}, \Gamma_{1}\left(x_{2 n-1}\right)\right)}\right) \\
& +\left(P _ { \alpha } ^ { 2 } \left(x^{\star}, \Gamma_{2}\left(x_{2 n-1}\right)\right.\right. \\
& \left.\left.\left.+\frac{P_{\alpha}^{2}\left(x^{\star}, \Gamma_{1}\left(x^{\star}\right)\right)+P_{\alpha}^{2}\left(x_{2 n-1}, \Gamma_{2}\left(x^{\star}\right)\right) P_{\alpha}^{2}\left(x_{2 n-1}, \Gamma_{2}\left(x_{2 n-1}\right)\right)}{1+P_{\alpha}^{2}\left(x^{\star}, \Gamma_{1}\left(x^{\star}\right)\right) P_{\alpha}^{2}\left(x_{2 n-1}, \Gamma_{2}\left(x^{\star}\right)\right) P_{\alpha}^{2}\left(x_{2 n-1}, \Gamma_{2}\left(x_{2 n-1}\right)\right)}\right)\right\}\right\} \\
& \leq q \operatorname{qax}\left\{\left\|x^{\star}-x_{2 n-1}\right\|^{2}, P_{\alpha}^{2}\left(x^{\star}, \Gamma_{1}\left(x^{\star}\right)\right), P_{\alpha}^{2}\left(x_{2 n-1}, x_{2 n}\right)\right), \\
& \left\{\frac{P_{\alpha}^{2}\left(x_{2 n-1}, \Gamma_{1}\left(x^{\star}\right)\right)+P_{\alpha}^{2}\left(x^{\star}, x_{2 n}\right)}{1+P_{\alpha}^{2}\left(x_{2 n-1}, \Gamma\left(x^{\star}\right)\right) P_{\alpha}^{2}\left(x^{\star}, x_{2 n}\right)}\right\}, \\
& \frac{1}{2}\left\{\left(P_{\alpha}^{2}\left(x^{\star}, \Gamma_{1}\left(x^{\star}\right)\right)+\frac{P_{\alpha}^{2}\left(x_{2 n-1}, \Gamma_{1}\left(x^{\star}\right)\right)+P_{\alpha}^{2}\left(x^{\star},\left(x_{2 n}\right)\right.}{1+P_{\alpha}^{2}\left(x_{2 n-1}, \Gamma_{1}\left(x^{\star}\right)\right) P_{\alpha}^{2}\left(x^{\star}, x_{2 n}\right)}\right)\right. \\
& \left.+\left(P_{\alpha}^{2}\left(x_{2 n-1}, x_{2 n}\right)+\frac{P_{\alpha}^{2}\left(x^{\star}, \Gamma_{1}\left(x^{\star}\right)\right)+P_{\alpha}^{2}\left(x_{2 n-1}, \Gamma_{1}\left(x^{\star}\right)\right)}{1+P_{\alpha}^{2}\left(x^{\star}, \Gamma_{1}\left(x^{\star}\right)\right) P_{\alpha}^{2}\left(x_{2 n-1}, \Gamma_{1}\left(x^{\star}\right)\right)}\right)\right\}, \\
& \frac{1}{2}\left\{\left(P_{\alpha}^{2}\left(x^{\star}, \Gamma_{1}\left(x^{\star}\right)\right)+\frac{P_{\alpha}^{2}\left(x^{\star}, x_{2 n}\right)+P_{\alpha}^{2}\left(x_{2 n-1}, \Gamma_{1}\left(x^{\star}\right)\right) P_{\alpha}^{2}\left(x_{2 n-1}, x_{2 n}\right)}{1+P_{\alpha}^{2}\left(x_{2 n-2}, x_{2 n}\right) P_{\alpha}^{2}\left(x_{2 n-1}, \Gamma_{1}\left(x^{\star}\right)\right) P_{\alpha}^{2}\left(x_{2 n-1}, x_{2 n}\right)}\right)\right. \\
& \left.\left.+\left(P_{\alpha}^{2}\left(x^{\star}, x_{2 n}\right)+\frac{P_{\alpha}^{2}\left(x^{\star}, \Gamma_{1}\left(x^{\star}\right)\right)+P_{\alpha}^{2}\left(x_{2 n-1}, \Gamma_{1}\left(x^{\star}\right)\right) P_{\alpha}^{2}\left(x_{2 n-1}, x_{2 n}\right)}{1+P_{\alpha}^{2}\left(x^{\star}, \Gamma_{1}\left(x^{\star}\right)\right) P_{\alpha}^{2}\left(x_{2 n-1}, \Gamma_{1}\left(x^{\star}\right)\right) P_{\alpha}^{2}\left(x_{2 n-1}, x_{2 n}\right)}\right)\right\}\right\},
\end{aligned}
$$

it follows (from the continuity of $P_{\alpha}$ ) that

$$
\left.P_{\alpha}^{2}\left(\Gamma_{1}\left(x^{\star}\right), x^{\star}\right)\right) \leq 2 q P_{\alpha}^{2}\left(x^{\star}, \Gamma_{1}\left(x^{\star}\right)\right)
$$


as $n \rightarrow \infty$. Since $q \in\left(0, \frac{1}{4}\right)$, it follows that $\left.P_{\alpha}^{2}\left(\Gamma_{1}\left(x^{\star}\right), x^{\star}\right)\right)=0$. Thus, by Lemma 2.5, we have $x^{\star} \subset \Gamma_{1} x^{\star}$. Using the same argument as above, we get $x^{\star} \subset \Gamma_{2} x^{\star}$. Consequently, $x^{\star} \subset \Gamma_{1} x^{\star} \cap \Gamma_{2} x^{\star}$. The proof of Theorem 3.2 is completed.

\section{Conclusion}

An affirmative answer has been provided for Question 1.1. The results obtained in this paper improve and generalise the corresponding results in [10], [9] and several others currently announced in literature.

\section{REFERENCES}

[1] S. Heilpern, Fuzzy mappings and fixed point theorem, J. Math. Anal. Appl. 83 (1981) 566?569. https: //doi.org/10.1016/0022-247X (81)90141-4.

[2] B. Soo Lee, S. Jin Cho, A fixed point theorem for contractive-type fuzzy mappings, Fuzzy Sets Syst. 61 (1994) 309?312. https://doi.org/10.1016/0165-0114(94)90173-2.

[3] R.K. Bose, D. Sahani, Fuzzy mappings and fixed point theorems, Fuzzy Sets Syst. 21 (1987) 53?58. https://doi.org/10.1016/0165-0114(87)90152-7.

[4] B.S. Lee, M.K. Kang, A generalisation of Som and Mukherjee's ?xed point theorem, Indian J. Math. 41 (1999) 205-209.

[5] B.E. Rhoades, Fixed points of some fuzzy mappings, Soochow J. Math. 22 (1996) 111-115.

[6] S.L. Singh, R. Talwar, Fixed points of fuzzy mappings, Soochow J. Math. 19 (1993), 95-102.

[7] B.E. Rhoade, A comparison of various definitions of contractive mappings, Trans. Amer. Math. Soc. 266 (1977), 257-290.

[8] T. Som, R.N. Mukherjee, Some fixed point theorems for fuzzy mappings, Fuzzy Sets Syst. 33 (1989) 213-219.

[9] P.N. Dutta, B.S. Choudhury, Fixed point of fuzzy mappings in Hilbert spaces, Math. Commun. 7 (2002), 91-96.

[10] A.S. Saluja, D. Macarde, P.K. Jhade, Fixed point of fuzzy mappings in Hilbert spaces, Asia Pac. J. Math. 1 (2014) 127-133.

[11] L.A. Zadeh, Fuzzy Sets, Inform. Control, 8 (1965) 338-353.

[12] R.D. Daheriya, U. Dongre, M. Ughade, Some common fixed point theorems for fuzzy maps under nonexpansive-type condition, Int. J. Sci. Innov. Math. Res. 4 (2016) 1-10.

[13] M. Gregus, A fixed point theorem in Banach spaces, Boll. Un. Mat. Ital. A, 5 (1980) 193-198.

[14] L. Ciric, On some nonexpansive type mappings and fixed points, Indian J. Pure Appl. Math. 24 (1993), 145-149.

[15] S. Banach, Sur les operations dans les ensemles abstraits et leur application aux equations integrales, Fund. Math. 3 (1922) 133-181.

[16] l. Beg, M. Abbas, Invariant approximation for fuzzy nonexpansive mappings, Math. Bohemica. 136 (2011) $51-59$. 